\title{
PROGRESS TOWARD AN ALL NIOBIUM SUPERCONDUCTING RF PHOTOCATHODE ELECTRON GUN*
}

\author{
M. Cole, H. Bluem, J. Rathke, T. Schultheiss, Advanced Energy Systems Inc, Medford, NY, USA \\ I. Ben-Zvi, T. Srinivasan-Rao, BNL, Upton, NY, USA
}

\begin{abstract}
Advanced Energy Systems Inc (AES) and Brookhaven National Laboratory (BNL) are currently working on a program to develop a Superconducting Photocathode Electron Gun using an all $\mathrm{Nb}$ cavity. The work is being performed as a Phase II SBIR and under a CRADA between AES and BNL. Results of initial efforts have been presented previously $[1,2]$. This paper will review the progress being made in this program. Results of revision and optimization of the $\mathrm{Nb}$ cavity will be shown. A brief overview of the thermal analysis being performed in support of this work will be included, a more detailed discussion will appear under a separate title [3]. The beam dynamics and anticipated beam performance will be discussed. An overview of the mechanical design of the cavity will be presented along with a discussion of, and figures showing, the current status of the fabrication process. Our approach to providing the cryostat in which the cavity will be tested will also be included along with a discussion of, and figures showing, the current status of the fabrication process. Finally, we will review our future plans which include the assembly and integration of the cryostat at AES and testing at BNL.
\end{abstract}

\section{QUANTUM EFFICIENCY MEASUREMENTS}

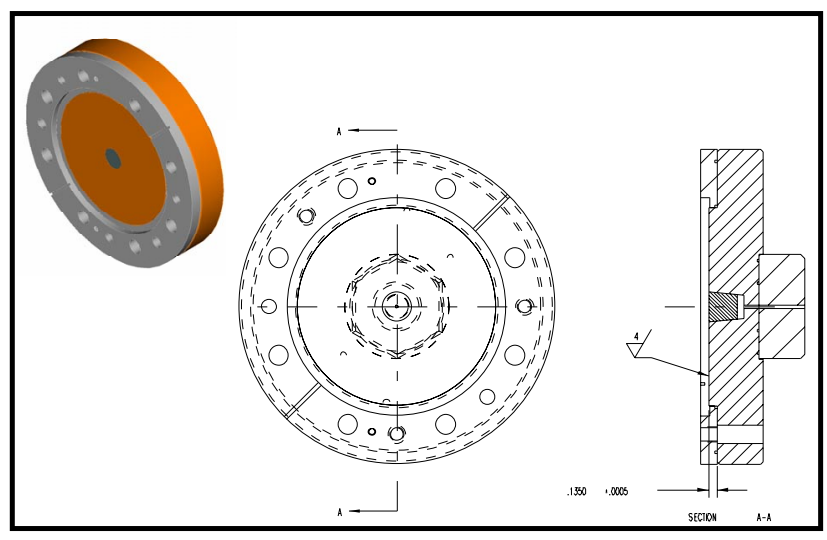

Figure 1 - ATF backplate with brazed $\mathrm{Nb}$ cathode

To further validate and understand the $\mathrm{QE}$ of the niobium cathode we have designed a replacement cathode

\footnotetext{
*Work supported by the U.S. DOE., Office of Basic Energy Sciences, under Contract Nos. DE-AC02-98CH10886DOE, the SBIR grant DEFG02-99ER82724, and under CRADA No. BNL-C-00-15. Such support does not constitute an endorsement by DOE of the views expressed in this paper.
}

plate for the BNL ATF electron gun. This plate will exactly duplicate the geometry of the current cathode but will incorporate a brazed niobium slug as the cathode. The method for fabrication calls for nickel plating the tapered niobium plug and brazing it in a tapered hole in the backplate using Au-Cu alloy. The backplate will then be finish machined to remove sufficient copper and niobium to expose virgin material. This surface will then be prepared via standard BNL cathode preparation techniques. Figure 1 shows the design of the cathode testing backplate.

We have designed a new fixture for testing of niobium cathode samples for quantum efficiency. One goal of the new series of tests will be to see if there is any measurable effect (positive or negative) associated with the process used to assemble the cathode test unit that will be tested at the ATF facility. The brazed assembly described previously will be used to fabricate several test cathodes for this purpose.

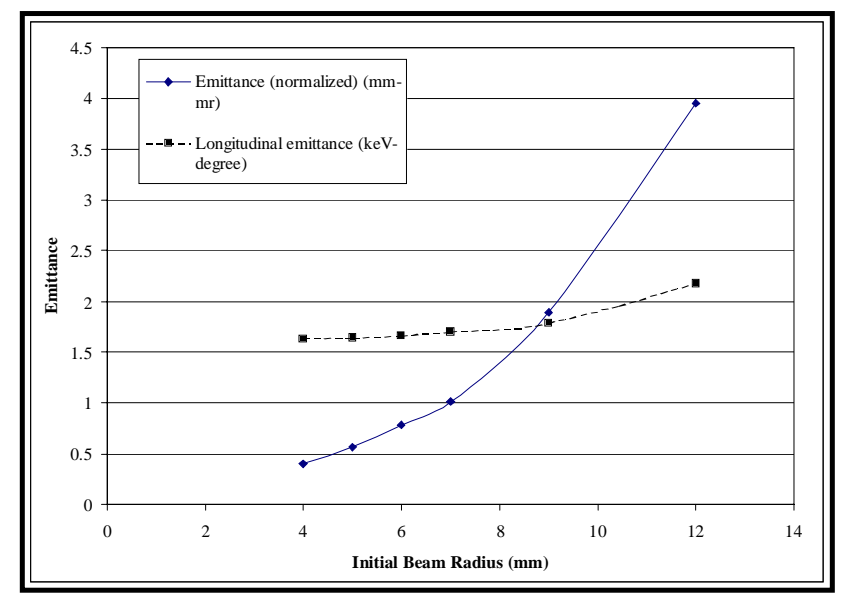

Figure 2 - Emittance scaling with beam radius

\section{BEAM DYNAMICS CALCULATIONS}

The first step taken in the beam dynamics studies of the revised cavity was a brief parameter space search. For these studies, the field gradient at the cathode surface was $35 \mathrm{MV} / \mathrm{m}$, the bunch charge was fixed at $0.01 \mathrm{nC}$, and the seed laser parameters set at a radius of $4.5 \mathrm{~mm}$ RMS with a pulse length of 6 ps FWHM. An emittance minimum between 45 and 50 became evident, while the energy spread continues decreasing. There is a certain trade-off to be made between the minimum transverse emittance and the minimum longitudinal emittance. It was found that a reduced pulse length at this low charge also 
provided better transverse and longitudinal emittance performance, but a complete study was not performed. Reducing the laser pulse length to 4 ps FWHM resulted in a $6 \%$ improvement in the transverse emittance and a $30 \%$ improvement in the longitudinal. The laser pulse length was not reduced further.

Following this, we checked the effect of the seed laser radius (this is the same as the initial electron beam radius). The radius was varied from $4 \mathrm{~mm}$ up to $12 \mathrm{~mm}$ as the change in the transverse and longitudinal emittance were observed. As expected, the emittance is an increasing function of the initial beam radius. The results are shown in figure 2

\section{CAVITY RF DESIGN}

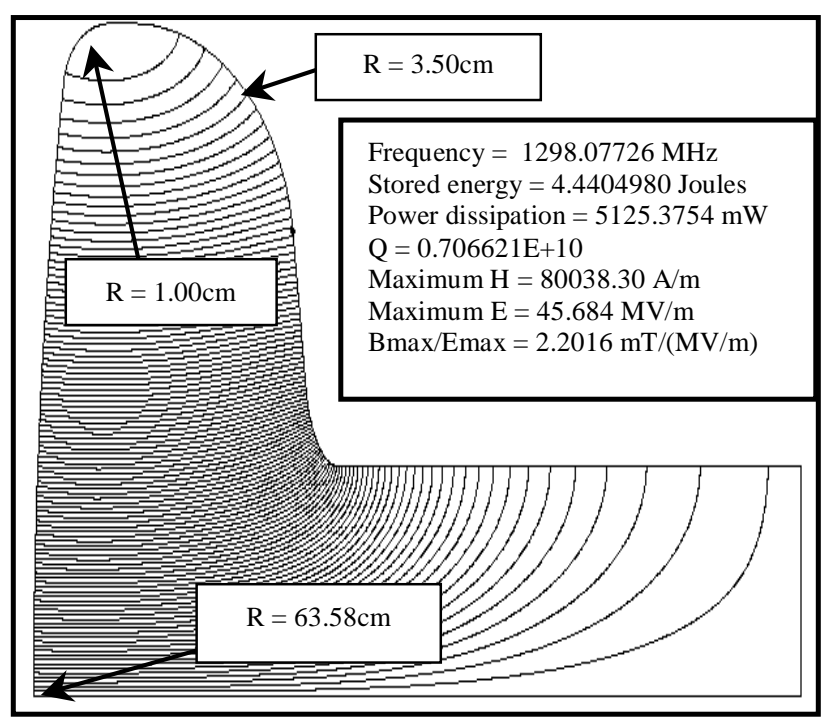

Figure 3 - Gun fields and RF parameters

The initial concept for this gun utilized a protruding $\mathrm{Nb}$ cathode on the backplate of the cavity. Previously we determined that there was no benefit to this approach [1]. Our current cavity design includes a slightly domed backplate. This configuration is shown in figure 3 . The dome shape adds stiffness to the cavity backplate and eliminates the requirement for additional stiffeners to be added.

\section{CAVITY THERMAL AND STRUCTURAL ANALYSIS}

The niobium cavity wall thickness is sized to operate with a 15 psi pressure load on the outside of the cavity and vacuum on the inside, pass 1 watt of laser power and the RF surface thermal load through its thickness while maintaining superconducting temperatures.

An axisymmetric model of the cavity was used to size the walls. The thermal analysis assumed $1.8 \mathrm{~K}$ superfluid helium on its outer surface. The heat flux through this surface $\left(\Phi_{\mathrm{s}}\right)$ is determined by the temperature dependent Kapitza conductivity $\mathrm{H}_{\mathrm{k}}\left(\mathrm{T}_{\mathrm{b}}, \Delta \mathrm{T}\right)$.

When $\Delta \mathrm{T}$ is greater than $1.4 \mathrm{~K}$, the surface flux exceeds the critical heat flux for helium II and the local

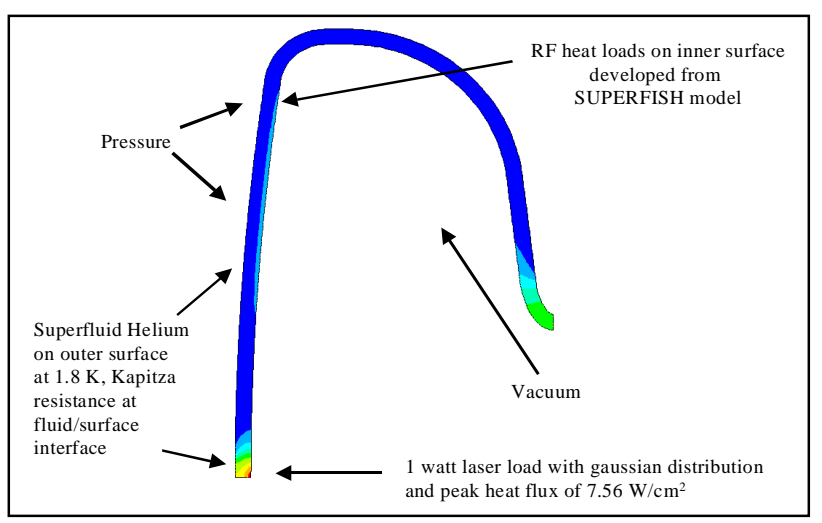

Figure 4 - Thermal model boundary conditions

conductivity is greatly reduced. The heat flow will then redistribute if the wall thickness is large enough.

Figure 4 shows the thermal model and the boundary conditions. This model was run for three different wall thicknesses. Initially the temperature decreases as the wall thickness increases, this is due to the heat redistributing and lowering the heat flux on the coolant surface. The nonlinearity of this effect means that ultimately at larger thickness the temperature would increase as the wall thickness increased. The three and one-half $\mathrm{mm}$ thick wall gives the best thermal and structural results.

\section{ANTICIPATED OPERATING PARAMETERS}

Based on the QEF measurements made to date, the RF design of the cavity and the ability of the cavity/cryogen system to remove heat deposited by the laser we have calculated a set of anticipated operating parameters. These parameters are shown in table 1 and are shown for a series of available laser powers. The driver for laser power is our budget. In our current program we can only afford to purchase a $150 \mathrm{~mW}$ laser, so our performance will be limited accordingly.

Table 1 - Anticipated Operating Parameters

\begin{tabular}{|r|r|r|r|r|r|r|r|r|}
\hline $\begin{array}{l}\text { Laser Spot D } \\
(\mathrm{cm})\end{array}$ & $\begin{array}{l}\text { Laser Area } \\
(\mathrm{cm})\end{array}$ & $\begin{array}{l}\text { Max Laser Power } \\
(\mathrm{W})(6 \mathrm{w} / \mathrm{cm} 2)\end{array}$ & $\begin{array}{l}\text { Ecathode } \\
(\mathrm{MV} / \mathrm{m})\end{array}$ & $\begin{array}{l}\text { Quantum } \\
\text { Efficiency }\end{array}$ & $\begin{array}{l}\text { Electron } \\
\text { current }(\mathrm{mA})\end{array}$ & $\begin{array}{l}\text { Epeak } \\
(\mathrm{MV} / \mathrm{m})\end{array}$ & $\begin{array}{l}\text { Eap erture } \\
(\mathrm{MV} / \mathrm{m})\end{array}$ & $\begin{array}{l}\text { Bunch Charge with } \\
81.25 \mathrm{MHz} \text { Laser }(\mathrm{nC})\end{array}$ \\
\hline 0.460659 & 0.167 & 0.150 & 43.76 & 0.002054 & 0.066125 & 45.85 & 45.85 & 0.81385 \\
\hline 0.460659 & 0.167 & 1.000 & 43.76 & 0.002054 & 0.440835 & 45.85 & 45.85 & 5.42566 \\
\hline 0.651469 & 0.333 & 2.000 & 43.76 & 0.002054 & 0.881653 & 45.85 & 45.85 & 10.85111 \\
\hline 1.000000 & 0.785 & 4.712 & 43.76 & 0.002054 & 2.077352 & 45.85 & 45.85 & 25.56741 \\
\hline
\end{tabular}




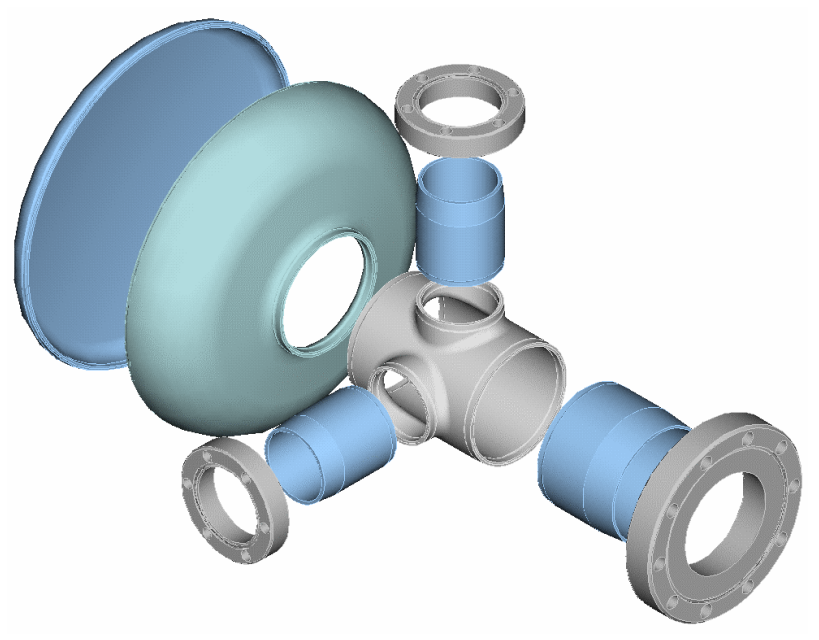

Figure 5-Exploded view of gun cavity

\section{CRYOSTAT DESIGN AND INTEGRATION}

The cryostat requirement for the superconducting gun will be met by modifying an existing cryostat that is available from BNL. We have moved the cryostat from BNL to AES and have begun the modification process. Figure 7 shows the cryostat along with a cutaway view of the superconducting gun installed in the cryostat.

The only required modification to the cryostat is pointed out in the figure as the replacement of the lower closure plate on the helium vessel. The existing vessel has a simple flat plate. We require added depth for the cavity to fit, however, even with the added depth, the existing nitrogen shield vessel can be used. The cavity will ultimately be installed with the coupler ports in a horizontal orientation and the cavity will be supported by a rigid beam tube connected to the vessel flange (not shown in figure). This requires the minimum amount of rework on the cryostat and the simplest cavity support.

\section{CAVITY MECHANICAL DESIGN}

The mechanical design of the gun cavity is largely complete. Figure 5 shows an exploded view of the cavity with the beam ports and tubes. A cold model of the cavity has been fabricated and is undergoing test. Fabricating the cold model provided an opportunity to prove out the fabrication of the solid model of the cavity shape. This process is similar to the steps which will be required to fabricate the tooling to be used to form the $\mathrm{Nb}$ cavity pieces. We are also fabricating pulled nipples for the coupling ports on the beam tube. The pulling of a test nipple is shown in figure 6 . The tooling and pulling process used will be quite similar to that to be used during the fabrication of the actual $\mathrm{Nb}$ parts.

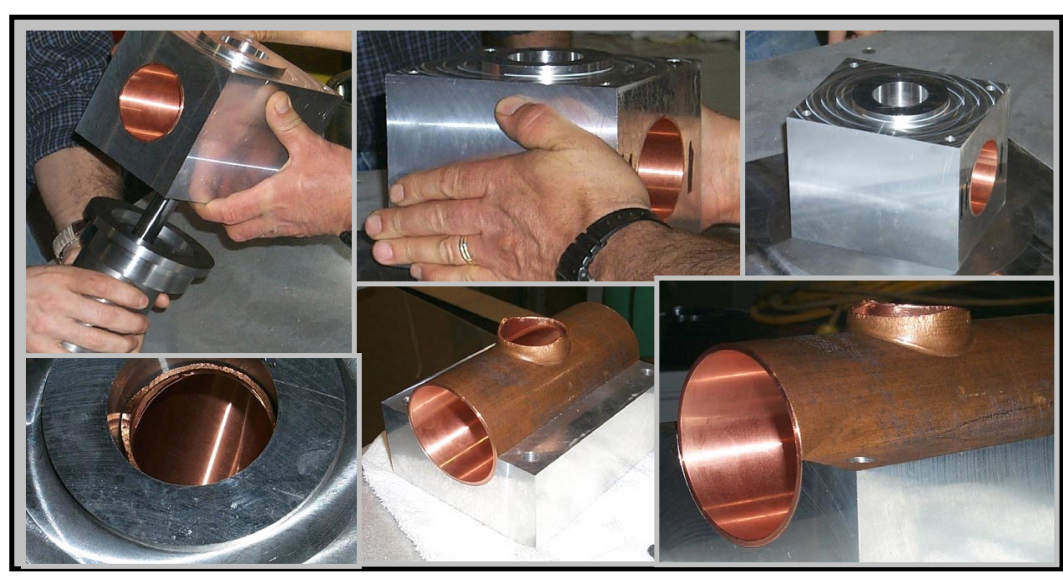

Figure 6 - Test pull of beam tube ports

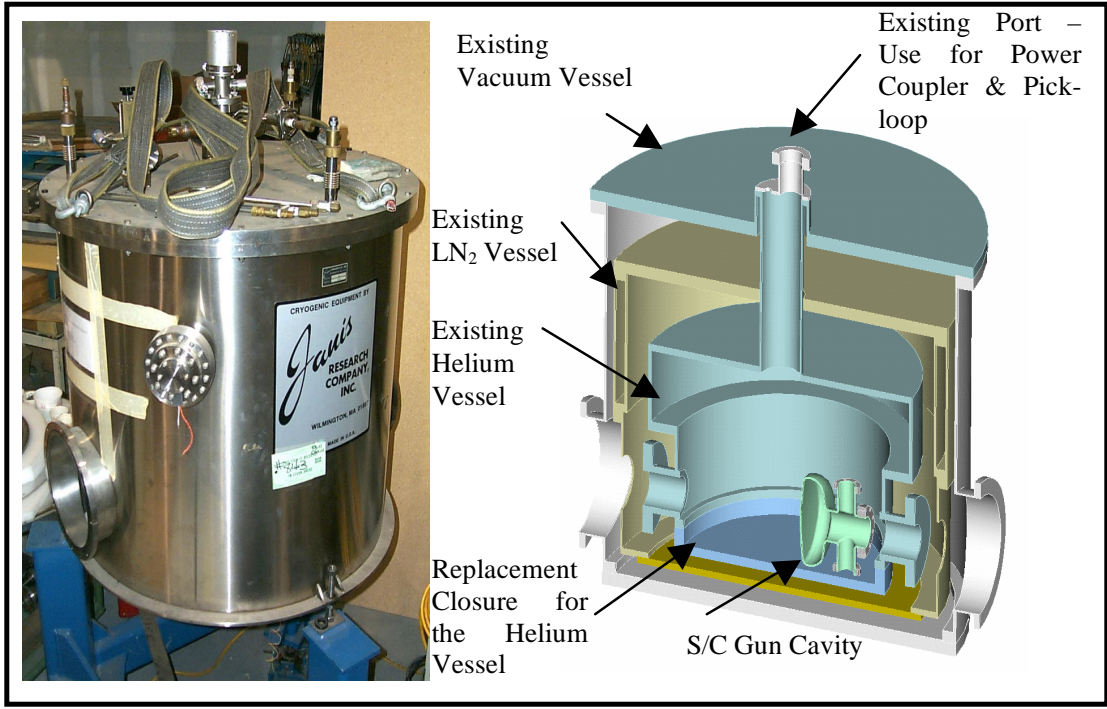

Figure 7 - Cryostat Photo and Cutaway View

\section{REFERENCES}

[1] "Development of a Novel Superconducting RF Photocathode Electron Gun", M. Cole, et. al., 2000 LINAC Conference in Monterey, California.

[2] "Thermal Structural Analysis of an SCRF Photocathode Electron Gun Cavity", T. SCHULTHEISS, et. al., 2000

LINAC Conference in Monterey,

California.

[3] "Thermal/Structural Analysis of an All Niobium Superconducting RF Photocathode Electron Gun", T. SCHULTHEISS, et. al., This Conference 